\title{
Cinobufacini promotes apoptosis of bladder cancer cells by influencing the expression of autophagy-related genes
}

\author{
DONG CHEN, JUNYI CHEN, YIHONG GUO and YINING LI \\ Department of Urology, The Second Affiliated Hospital of Fujian Medical University, \\ Quanzhou, Fujian 362000, P.R. China
}

Received November 24, 2017; Accepted January 22, 2018

DOI: $10.3892 / \mathrm{ol} .2018 .8206$

\begin{abstract}
The effects of cinobufacini on apoptosis of human bladder cancer T24 cells and the expression of autophagy-related genes and proteins were studied. The human bladder cancer T24 cells were selected, and the inhibitory effect of cinobufacini on the proliferation of human bladder cancer cells was detected by cell viability assay. Flow cytometry and Hoechst staining were used to detect the changes in the apoptosis of bladder cancer cells after being treated with cinobufacini; the changes in the expression levels of human bladder cancer cell apoptosis-related genes and proteins, cleaved caspase-3, Bax, B-cell lymphoma-2 (Bcl-2) and autophagy-related genes and proteins, p62, light chain 3 (LC3) and autophagy-related protein 7 (Atg7) after treatment with cinobufacini were detected by western blot analysis and reverse transcription polymerase chain reaction (RT-PCR). 3-(4,5-dime thyl-2-thiazolyl)-2,5-diphenyl-2-H-tetrazolium bromide (MTT) assay. The results showed that $0.1 \mathrm{mg} / \mathrm{ml}$ cinobufacini significantly inhibited the proliferation of human bladder cancer cells cultured in vitro $(\mathrm{P}<0.01)$, and it was dose- and time-dependent. Both flow cytometry and Hoechst staining showed that cinobufacini promoted the apoptosis of cells $(\mathrm{P}<0.01)$, and the level of cell apoptosis increased with the increase of drug concentration. Both western blot analysis and RT-PCR showed that cinobufacini could decrease the expression level of Bcl-2 in T24 cells $(\mathrm{P}<0.01)$, increase the expression levels of Bax and cleaved caspase- $3(\mathrm{P}<0.01)$, increase the ratio of Bax/Bcl-2 $(\mathrm{P}<0.01)$, upregulate the expression level of the angiotensin-related protein p62 $(\mathrm{P}<0.01)$, reduce the ratio of LC3-II/I $(\mathrm{P}<0.01)$ and decrease the expression level of Atg7 $(\mathrm{P}<0.01)$. After treatment with rapamycin, the expression levels of Bcl-2, Bax, cleaved caspase-3, autophagy-related genes
\end{abstract}

Correspondence to: Dr Yining Li, Department of Urology, The Second Affiliated Hospital of Fujian Medical University, 34 North Zhongshan Avenue, Licheng, Quanzhou, Fujian 362000, P.R. China E-mail: yiningli_001@163.com

Key words: bladder cancer, autophagy, cinobufacini, apoptosis and proteins, p62, LC3-II/I and Atg7 were similar to those in the control group. Cinobufacini can inhibit the autophagy activation of bladder cancer cells, thus promoting apoptosis of bladder cancer T24 cells and inhibiting the proliferation of T24 cells, which may provide a theoretical basis for the development of new anti-bladder cancer drugs.

\section{Introduction}

Bladder cancer is the most common malignant tumor in the urinary system, which often occurs in the bladder mucosa. According to the statistics of the World Health Organization (WHO), bladder cancer is one of the world's top ten common tumors $(1,2)$. Bladder urothelial carcinoma is divided into non-muscular and myometrial infiltrating urothelial carcinoma. Patients with non-muscular infiltrating urothelial carcinoma are often treated with transurethral resection of bladder tumor, and intravesical instillation therapy after operation is used to prevent recurrence (3). Metastatic bladder cancer is mainly treated with chemotherapy, and the maximal effective rate of chemotherapy can reach $65 \%$ (4). Cinobufacini is made from the refined fat-soluble components in the dried toad skin extracted using a scientific method, which contains toad aglucones and bufalin that have an extraordinary anticancer effect, and the anticancer activity of the ingredients is ten times or even a hundred times stronger than those of the currently clinically used anticancer drugs such as paclitaxel, doxorubicin and camptothecin with no side effects, bringing benefits to many cancer patients $(5,6)$. Oral administration of cinobufacini has good curative effects in the treatment of liver, stomach, lung, colon, esophageal, pancreatic cancer and acute leukemia (6-9). However, there is no study on the effect of cinobufacini on human bladder cancer. Autophagy is a lysosomal-dependent degradation way in eukaryotic cells, which produces amino acids, fatty acids and adenosine triphosadenine for cell re-use by degrading the injured organelle proteins under hypoxia and other stress conditions, it is the survival mechanism of cells (including tumor cells) under the harsh environment (10), and it is also one of the mechanisms of drug resistance of many tumor cells.

The purpose of the present study was to elucidate the value of cinobufacini for bladder cancer by investigating the effect of cinobufacini on human bladder cancer cell line T24, its effect on T24 cell apoptosis and its specific mechanism, 
thus providing a theoretical basis for the clinical treatment of bladder cancer.

\section{Materials and methods}

Instruments and materials. Human bladder cancer cell line T24 was purchased from the Cell Bank, Shanghai Institute of Life Sciences, Chinese Academy of Sciences (Shanghai, China); 3-(4,5-dimethyl-2-thiazolyl)-2,5-diphenyl-2-H-tetrazolium bromide (MTT) kits (R\&D Systems, Inc., Minneapolis, MN, USA), dimethyl sulfoxide (DMSO), cinobufacini standards and rapamycin (Sigma-Aldrich; Merck \& Co., Inc., Whitehouse Station, NJ, USA); rabbit anti human polyclonal Bcl-2 (dilution, 1:500; cat. no. 2872), Bax (dilution, 1:500; cat. no. 2774), cleaved caspase-3 (dilution, 1:500; cat. no. 9661), p62 (dilution, 1:500; cat. no. 5114), LC3B (dilution, 1:500; cat. no. 2775) and Atg7 (dilution, 1:500; cat. no. 2631) antibodies were purchased from Cell Signaling Technology, Inc., (Danvers, MA, USA). Rabbit anti-human polyclonal GAPDH antibody (dilution, 1:500; cat. no. SAB2100894) was purchased from Sigma Aldrich; Merck \& Co. Inc. Horseradish peroxidase-labeled goat anti-rabbit secondary polyclonal antibodies (dilution, 1:2,000; cat. no. 7074) was from Cell Signaling Technology, Inc.. Enhanced chemiluminescence (ECL) liquid (Invitrogen; Thermo Fisher Scientific, Inc., Waltham, MA, USA), pipettes (Eppendorf, Hamburg, Germany), polymerase chain reaction (PCR) instruments (Applied Biosystems; Thermo Fisher Scientific, Inc.), ultraviolet imaging system (Biometra GmbH, Göttingen, Germany), electronic balance (BP121S; Sartorius AG, Göttingen, Germany), $-80^{\circ} \mathrm{C}$ refrigerator and low temperature centrifuge (Thermo Fisher Scientific, Inc.); microplate reader (Bio-Rad Laboratories, Inc., Hercules, CA, USA). The study was approved by the Ethics Committee of The Second Affiliated Hospital of Fujian Medical University (Quanzhou, China).

Cell culture methods. The medium was replaced on an ultra-clean workbench for the human bladder cancer cell line T24 after the purchase. Cells were further cultured using Dulbecco's modified Eagle's medium (DMEM) (ultra-high glucose; containing $10 \%$ inactivated fetal bovine serum and $1 \%$ streptomycin) in an incubator with $5 \% \mathrm{CO}_{2}$ and saturated humidity at $37^{\circ} \mathrm{C}$ until the cells grew to about $80 \%$ of the whole culture flask, and then they were subcultured. After that, the subcultured cells were further cultured under the above conditions until the cells grew again to the logarithmic growth phase, and the cells were treated. Plates were paved for follow-up experiments.

Detection of cell viability. Human bladder cancer T24 cells in the logarithmic growth phase were prepared into $4 \times 10^{6}$ single cell suspension and inoculated in 96-well plates at $100 \mu \mathrm{l} /$ well. After the cells were cultured in an incubator with $5 \% \mathrm{CO}_{2}$ and saturated humidity at $37^{\circ} \mathrm{C}$ for $24 \mathrm{~h}, 0.05,0.1$ and $0.5 \mathrm{mg} / \mathrm{ml}$ cinobufacini were used for treatment (cinobufacini was dissolved with $0.5 \%$ DMSO and diluted with medium), respectively. A total of six repeated wells were set in each group, and the DMSO treatment group was regarded as the negative control group. After the drug acted on the cells for a certain period of time, the cell viability detection test was performed according to the procedures of the MTT reagent instructions. After the cell culture was terminated, MTT-labeled reagent was added into the culture plates at $10 \mu \mathrm{l} /$ well (the final concentration was $0.5 \mathrm{mg} / \mathrm{ml}$ ). The plates were placed in an incubator with $5 \% \mathrm{CO}_{2}$ for incubation at $37^{\circ} \mathrm{C}$ for $4 \mathrm{~h}$. Then the dissolving solution was added into plates at $100 \mu \mathrm{l} /$ well, which were placed in an incubator with $5 \% \mathrm{CO}_{2}$ at $37^{\circ} \mathrm{C}$ for incubation overnight. Afterwards, the optical density of each well was detected with $550 \mathrm{~nm}$ as the detection wavelength and $750 \mathrm{~nm}$ as the reference wavelength. According to the formula, cell proliferation inhibition rate $(\%)=(1-$ average $\mathrm{A}$ value in the medication well / average A value in the control well) $\times 100 \%$. The half maximal inhibitory concentration of cells (the concentration required for inhibiting the growth of $50 \%$ of cells; $\mathrm{IC}_{50}$ ) were calculated using the GraphPad Prism 6.0 (GraphPad Software, Inc. La Jolla, CA, USA) based on the effects of different drug concentrations on the inhibition rate of cell growth.

Detection of cell apoptosis rate by Annex V/propidium iodide (PI) double staining. T24 cells were collected after they grew to the logarithmic growth phase and then prepared into $8 \times 10^{6}$ single cell suspension. The cells were inoculated in 6-well plates with $300 \mu \mathrm{l}$ each and cultured in an incubator with 5\% $\mathrm{CO}_{2}$ and saturated humidity at $37^{\circ} \mathrm{C}$ for $24 \mathrm{~h}$, after which cinobufacini at different concentrations was added for treatment for $24 \mathrm{~h}$. The cells in each well were digested and washed with pre-cooled phosphate-buffered saline (PBS) three times, and then the above cell suspension was transferred to a flow tube. Annexin V-fluorescein isothiocyanate and PI were added to each tube, tubes were gently whirled, and the cells were incubated at room temperature for $15 \mathrm{~min}$. A cell flow cytometer was used to detect the cells, and the detection in all the groups needed to be finished within $1 \mathrm{~h}$, otherwise it would affect the detection effect.

Hoechst 33258 staining. T24 cells in the logarithmic growth phase were digested and prepared into single cell suspension. The cells at the density of $6 \times 10^{6}$ were inoculated in 24 -well plates and further incubated for $12 \mathrm{~h}$ in an incubator until they were in good growth condition, and then cinobufacini at different concentrations was added. At $24 \mathrm{~h}$ after the further culture, the upper culture solution was carefully discarded, and pre-cooled PBS was used to wash cells twice. After that, cells were fixed with $4 \%$ paraformaldehyde for $10 \mathrm{~min}$ and washed with distilled water 3 times with 5 min each time. After the washing, Hoechst 33258 staining solution was added for staining away from light for $10 \mathrm{~min}$. At the end of the staining, cells were washed with distilled water twice, after which the morphology of cells was observed under a microscope, and the level of cell apoptosis in each group was calculated.

Quantitative real-time PCR.Cinobufacini $(0.5 \mathrm{mg} / \mathrm{ml})$ was used to study the effects of drugs on apoptosis and autophagy-related genes. The control, cinobufacini $(0.5 \mathrm{mg} / \mathrm{ml})$ and rapamycin group $(0.5 \mathrm{mg} / \mathrm{ml}$ cinobufacini $+50 \mathrm{nM}$ rapamycin $)$ were set, and cells treated in the above groups were digested and washed with PBS 3 times. TRIzol reagents (TRIzol kit; Thermo Fisher Scientific, Inc.) were added, followed by the addition of 
Table I. Primer sequences of each gene.

\begin{tabular}{ll}
\hline Genes & \multicolumn{1}{c}{ Sequence } \\
\hline Bax & F: 5'-CTGACGGCAACTTCAACTGG-3' \\
& R: 5'-GTGAGGAGGCTTGAGGAGTC-3' \\
Bcl-2 & F: 5'-TGTGTGTGGAGAGCGTCAAC -3' \\
& R: 5'-GCCAGAGAAATCAAACAGAGG-3' \\
p62 & F: 5'-AGCTGCCCTCAGCCCTCTA -3' \\
& R: 5'-GGCTTCTCTTCCCTCCATGTT-3' \\
LC3 & F: 5'-AACATGAGCGAGTTGGTCAAG -3' \\
& R: 5'-GCTCGTAGATGTCCGCGAT-3' \\
Cleaved & F: 5'-TCACCATTCGGTCAATCAGAGC -3' \\
caspase-3 & R: 5'-ACCAAGGGAGAACCAGGAAACG-3' \\
GAPDH & F: 5'-ACCCACTCCTCCACCTTTGAC -3' \\
& R: 5'-TCCACCACCCTGTTGCTGTAG-3'
\end{tabular}

LC3, light chain 3; GAPDH, rabbit anti-glyceraldehyde-3-phosphate dehydrogenase; Bcl-2, B-cell lymphoma-2.

chloroform for centrifugation. The supernatant was obtained, and then isopropanol was added to extract cell RNAs in each group, after which RNAs were treated with $75 \%$ ethanol. After that, ethanol was discarded by centrifugation, and RNAs were dried. Then $50 \mu \mathrm{l}$ diethyl pyrocarbonate was added to obtain RNA samples of each group. The complementary DNA chain was obtained by reverse transcription using the reverse transcription PCR kit and was taken as the template, after which primers, Taq polymerase and buffer, deoxy-ribonucleoside triphosphate mixture and double distilled water were added to perform PCR amplification on the PCR instrument. Finally, the products were placed on a quantitative PCR instrument to determine the messenger RNA (mRNA) expression of target genes. The primers were synthesized by Tiangen Biotech Co., Ltd. (Beijing, China). The primer sequences of each gene are shown in Table I and Bax/GAPDH, Bcl-2/GAPDH, cleaved caspase-3/GAPDH, p62/GAPDH, LC3/GAPDH and
LC3/GAPDH were used to measure the expression level of related genes in each group.

Western blot analysis detection. The control, cinobufacini $(0.5 \mathrm{mg} / \mathrm{ml})$ and rapamycin group $(0.5 \mathrm{mg} / \mathrm{ml}$ cinobufacini $+50 \mathrm{nM}$ rapamycin) were set. Cells treated with the above-mentioned drugs were digested, and the lysate was added, followed by the centrifugation for $10 \mathrm{~min}$ at $1,200 \mathrm{x} g$ at $4^{\circ} \mathrm{C}$. The supernatant was the corresponding total protein in each group. The total protein concentration was measured using the bicinchoninic acid protein assay kit. Sodium dodecyl sulfate polyacrylamide gel electrophoresis was conducted for samples, which were then transferred to a polyvinylidene fluoride membrane (IPVH00010; Millipore Corp., Billerica, MA, USA). After that, Bax, Bcl-2, cleaved caspase-3, p62, LC3, Atg7 and GAPDH primary antibodies (dilution, 1:1,000) were used for incubation at $4^{\circ} \mathrm{C}$ overnight. After the washing, secondary antibodies conjugated with horseradish peroxidase (dilution, 1:5,000) were used for incubation for $1 \mathrm{~h}$. Then ECL mixture was added, and the tabletting time was determined according to the fluorescence intensity of the protein band. The fixation was conducted after color development, bands were scanned, and ImageJ software (National Institutes of Health, Bethesda, MD, USA) was used for gray value analysis.

Statistical analysis. The data of this study are expressed as mean \pm standard deviation. The data were analyzed by SPSS 19.0 software (SPSS Inc., Chicago, IL, USA), and the t-test was used for intergroup comparisons. Enumeration data were compared by Chi-square test, and intergroup comparisons were detected using analysis of variance (ANOVA) followed by post hoc test (least significant difference). The analysis of homogeneity variance showed that if the variance was homogeneous, pairwise comparisons were conducted using Bonferroni method; if the variance was not homogeneous, pairwise comparisons were conducted using Welch method. Multiple comparisons were conducted using Dunnett's T3 method. $\mathrm{P}<0.05$ was considered to indicate a statistically significant difference.
A

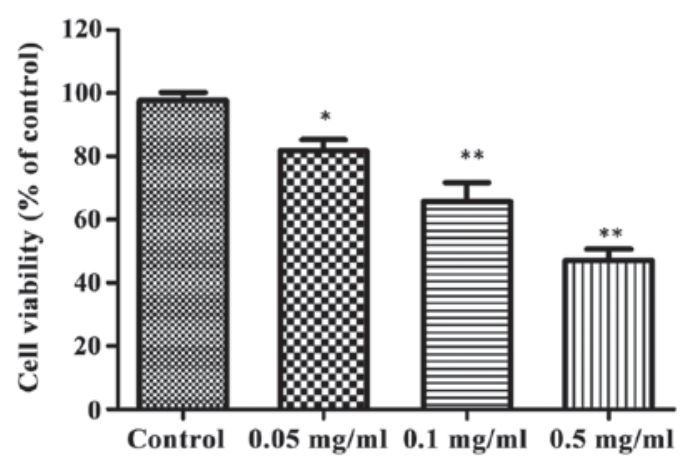

B

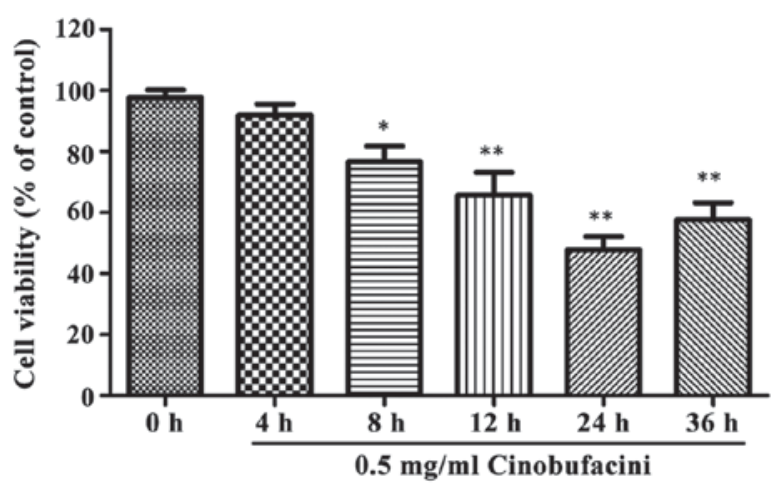

Figure 1. Detection of cell viability by MT assay. (A) Effects of cinobufacini at different concentrations on cell viability. (B) Effects of different treatment time on cell viability. After the treatment with cinobufacini for $24 \mathrm{~h}, 0.05,0.1$ and $0.5 \mathrm{mg} / \mathrm{ml}$ cinobufacini can significantly reduce the cell viability. ${ }^{*} \mathrm{P}<0.05$, ${ }^{* *} \mathrm{P}<0.01$. Cinobufacini $(0.5 \mathrm{mg} / \mathrm{ml})$ was used to treat cells for different time periods. At $24 \mathrm{~h}$ after treatment, the cell viability was the lowest, which is significantly lower than that of the control group, ${ }^{* *} \mathrm{P}<0.01$. 


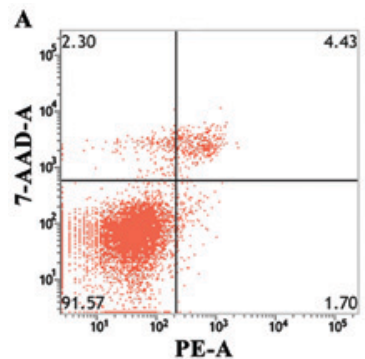

D

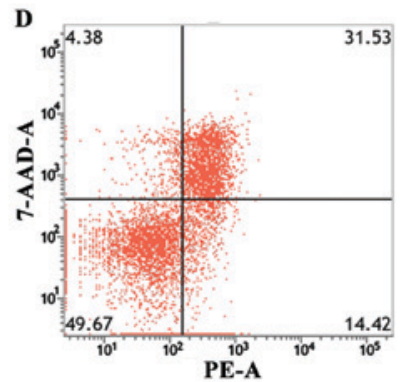

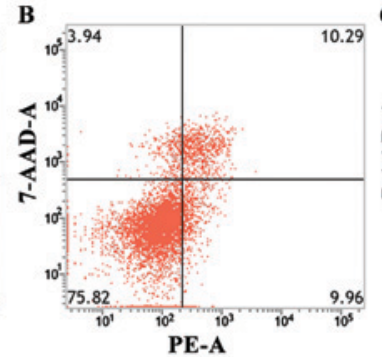

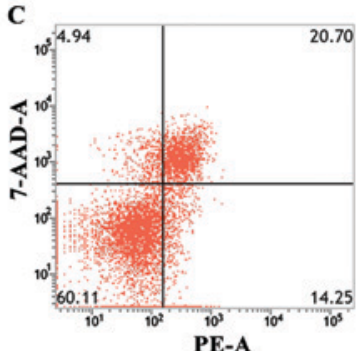

E
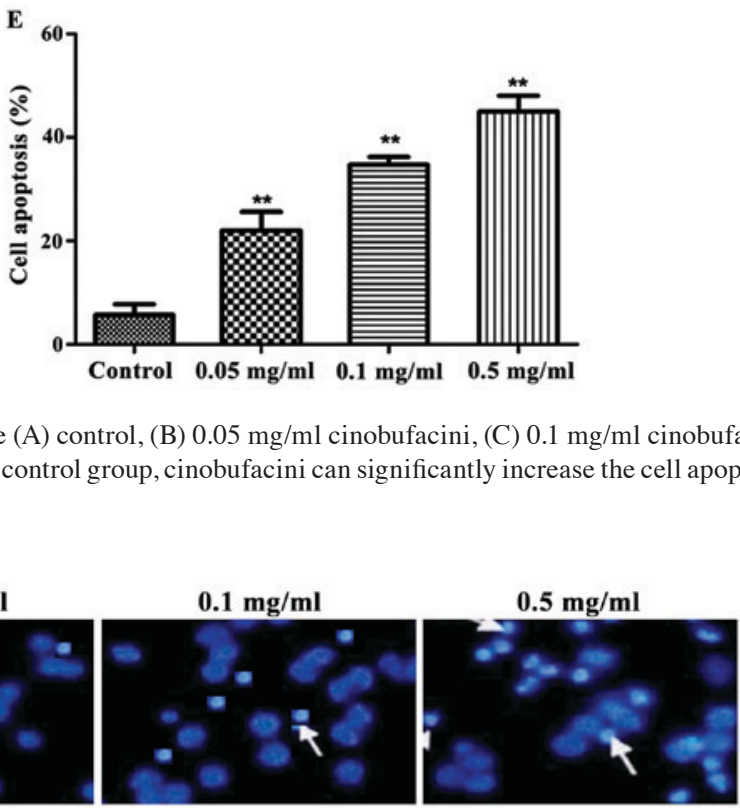

$\mathbf{B}$

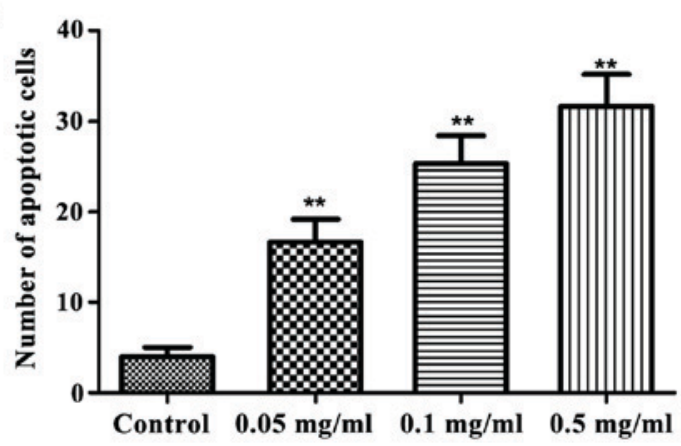

Figure 3. Detection of apoptosis by Hoechst 33258. (A) Hoechst staining of each group and (B) statistical graph. Compared with the control group, cinobufacini can significantly increase the apoptosis level of cells, ${ }^{* *} \mathrm{P}<0.01$.

\section{Results}

Detection of cell viability by MTT assay. MTT assay was used to detect the effects of cinobufacini at different concentrations on the viability of T24 cells. As shown in Fig. 1, after cells were treated with $0.05,0.1$ and $0.5 \mathrm{mg} / \mathrm{ml}$ cinobufacini for $24 \mathrm{~h}$, respectively, the cell viability was significantly decreased $(\mathrm{P}<0.05, \mathrm{P}<0.01)$. The effect of $0.5 \mathrm{mg} / \mathrm{ml}$ cinobufacini on cell viability at different time was different and time-dependent, and $24 \mathrm{~h}$ after the treatment, the cell viability was the lowest, which was significantly lower than that of the control group $(\mathrm{P}<0.01)$.

Detection of cell apoptosis by flow cytometer. Apoptosis of cells was measured by a flow cytometer. As shown in Fig. 2, the treatment with $0.05,0.1$ and $0.5 \mathrm{mg} / \mathrm{ml}$ cinobufacini could increase the apoptosis level of cells. The apoptosis level was the highest when the concentration of cinobufacini was $0.5 \mathrm{mg} / \mathrm{ml}(\mathrm{P}<0.01)$.

Detection of cell apoptosis by Hoechst staining. The effects of cinobufacini on apoptosis of human bladder cancer cell line T24 was detected by Hoechst 33258. As shown in Fig. 3, cinobufacini significantly increased the amount of apoptotic cells $(\mathrm{P}<0.01)$.

$m R N A$ expression level. The relative expression levels of apoptosis- and autophagy-related genes were detected by real-time quantitative PCR instrument. As shown in Fig. 4, after administration of cinobufacini, the expression levels of apoptosis-related genes, Bax and cleaved caspase- 3 in T24 cells were increased $(\mathrm{P}<0.01)$, that of $\mathrm{Bcl}-2$ was decreased $(\mathrm{P}<0.01)$, that of autophagy-related protein $\mathrm{p} 62$ was upregulated 
A

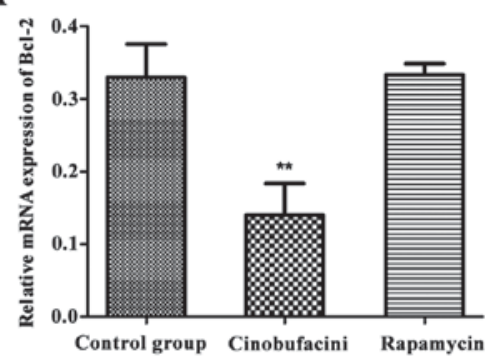

B

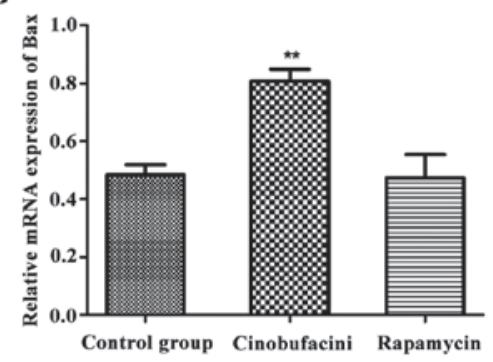

C

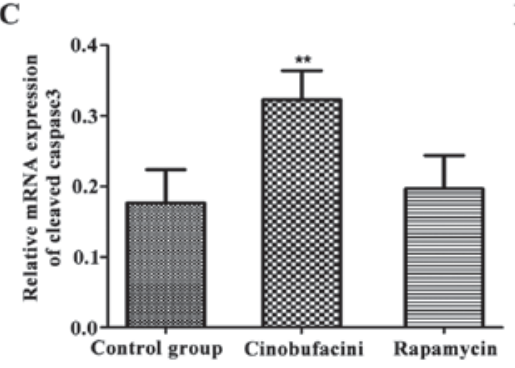

D

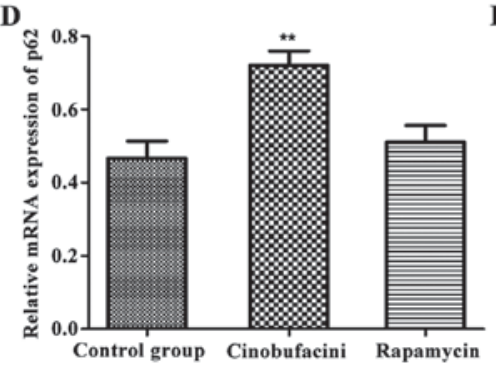

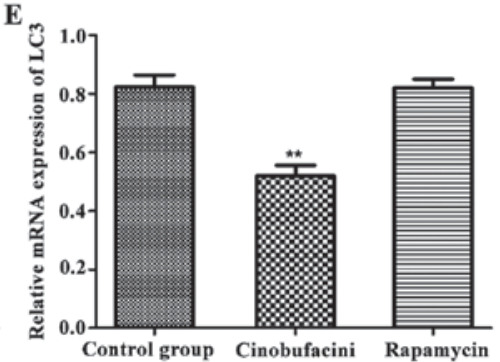

Figure 4. Detection of the expression levels of apoptosis- and autophagy-related genes in cells of each group by real-time quantitative PCR. Compared with those in the control group, the expression level of Bcl-2 is decreased, those of Bax and cleaved caspase- 3 are increased, that of p62 is upregulated and that of Atg7 is decreased. The expression levels of these genes are similar to those in the control group after administration of rapamycin. ${ }^{* *} \mathrm{P}<0.01$, compared with control group. PCR, polymerase chain reaction; Bcl-2, B-cell lymphoma-2; Atg7, autophagy-related protein 7.

A

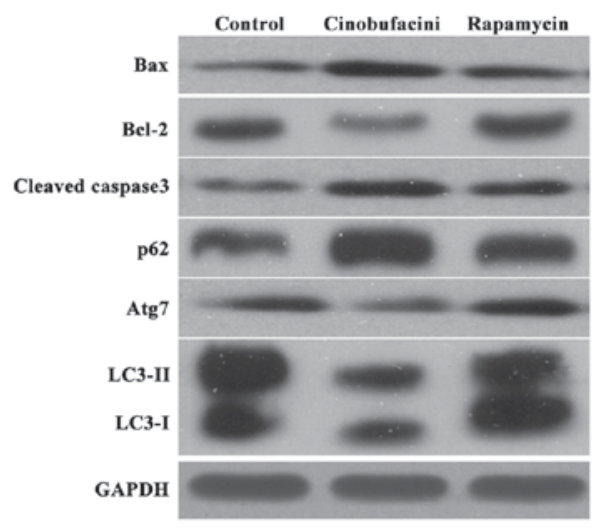

B

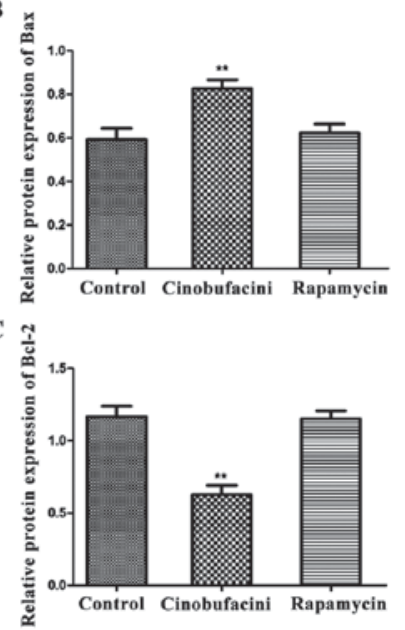

G
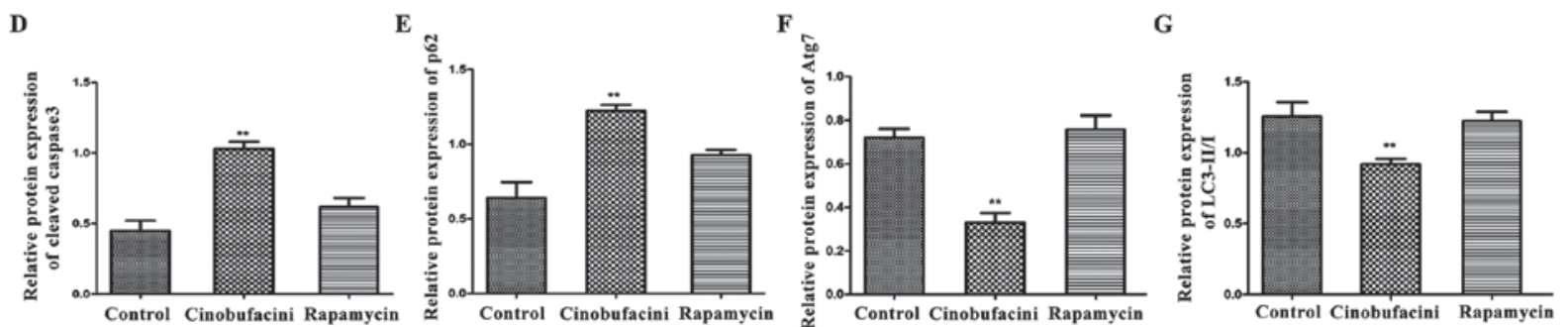

Figure 5. Detection of the expression levels of apoptosis- and autophagy-related proteins in each group by western blot analysis. (A) Protein band graphs of each group; and (B-G) statistical graph of each protein band. Compared with those in the control group, the expression level of Bcl-2 is decreased, those of Bax and cleaved caspase-3 are increased, that of p62 is upregulated, that of Atg7 is decreased, and the ratio of LC3-II/I is reduced, indicating that after administration of rapamycin, above changes in proteins can be reversed and the protein expression level is similar to that in the control group, ${ }^{* *} \mathrm{P}<0.01$. Bcl-2, B-cell lymphoma-2; Atg7, autophagy-related protein 7; LC3, light chain 3.

$(\mathrm{P}<0.01)$, and that of LC3 was decreased $(\mathrm{P}<0.01)$ compared with those in the control group. Protein changes caused by cinobufacini in the above conditions could be reversed after administration of rapamycin.
Protein expression level. Western blot analysis was used to detect the expression levels of apoptosis- and autophagy-related proteins in each group. As shown in Fig. 5, after administration of cinobufacini, the expression levels of apoptosis-related 
proteins, Bax and cleaved caspase- 3 in T24 cells were significantly increased $(\mathrm{P}<0.01)$, and that of $\mathrm{Bcl}-2$ was decreased $(\mathrm{P}<0.01)$ compared with those in the control group. After administration of rapamycin, the expression levels of Bcl-2, Bax and cleaved caspase-3 were comparable to those in the control group. After administration of cinobufacini, the expression levels of the autophagy-related protein p62 was upregulated $(\mathrm{P}<0.01)$, the ratio of LC3-II/I was decreased $(\mathrm{P}<0.01)$, and the expression level of Atg7 was decreased $(\mathrm{P}<0.01)$, indicating that rapamycin can reverse the above-mentioned changes induced by cinobufacini.

\section{Discussion}

Surgery combined with chemotherapy can significantly reduce the recurrence and deterioration of bladder cancer, but the current clinically commonly used chemotherapy drugs such as paclitaxel have a strong toxicity and irritation, limiting the treatment of patients and affecting the prognosis, thus reducing the effective rate of the treatment for bladder cancer $(11,12)$. Cinobufacini, as a traditional Chinese medicine preparation made from the toad skin, has been found to have significant heat clearing, detoxification, stasis and disintegrating mass effects, and it has less toxicity and irritation to patients (13). At present, a large number of research data show that cinobufacini significantly inhibits the proliferation of a variety of tumor cells and induces apoptosis (14). Ye et al (15) found that cinobufacini can block the cycle of leukemia cell lines, thus inducing the expression of apoptotic proteins and leading to cell apoptosis so as to inhibit cell proliferation. Wu et al (16) found that cinobufacini promotes apoptosis of liver cancer cells to a certain degree, but the mechanism still needs to be further studied. In the present study, it was found that cinobufacini could effectively inhibit the proliferation of bladder cancer cells and was dose- and time-dependent to some extent. Results of the flow cytometer and Hoechst staining revealed that cinobufacini could significantly increase the apoptosis level of bladder cancer cells. The above results suggested that the effect of cinobufacini on the proliferation of bladder cancer cells may be achieved by increasing apoptosis. Quantitative real-time PCR and western blot analysis were used to detect the expression levels of apoptosis- and autophagy-related proteins, respectively, which revealed that after the treatment with cinobufacini, the expression level of Bcl-2 was significantly decreased, while those of Bax and cleaved caspase- 3 were significantly increased, indicating cinobufacini can induce the occurrence of autophagy, that is, this can further confirm that the antitumor effect of cinobufacini is achieved by promoting apoptosis. The above results are consistent with the mechanism of antitumor effect of cinobufacini in pancreatic cancer found by Yin et al (17). The increased Bcl-2 expression level can inhibit apoptosis of cells, while the increased Bax expression level can promote apoptosis, which is achieved by inhibiting the activity of Bcl-2 (18).

Autophagy plays an important role in maintaining cell homeostasis, removing excess or necrotic organelles and so on. In previous years, it was found that the activation or inhibition of autophagy has important significance for the survival of tumor cells (19). In the present study, it was found that after the treatment with $0.5 \mathrm{mg} / \mathrm{ml}$ cinobufacini, the expression level of autophagy-related gene p62 was increased and that of Atg7 was decreased in the bladder cancer cell line T24. Western blot analysis showed that the expression level of autophagy-related protein p62 was increased, the ratio of LC3-II/I was decreased, and the expression level of Atg7 was decreased. The above results suggested that cinobufacini can effectively reduce the level of autophagy in bladder cancer cells and reduce the occurrence of autophagy. After administration of rapamycin, the levels of the above autophagy- and apoptosis-related proteins were reversed, which were comparable to those of the control group. Apoptosis of bladder cancer cells induced by cinobufacini might be caused by the autophagy pathway. The inhibition of autophagy by cinobufacini could significantly reduce the expression of $\mathrm{Bax}$, which led to the increased expression of Bcl-2, thus increasing the death of tumor cells.

In conclusion, cinobufacini can effectively reduce the survival rate and promote apoptosis of bladder cancer cells. Its mechanism may be to regulate the occurrence of apoptosis through the inhibition of autophagy; cinobufacini has the potential to be developed into a drug for the treatment of bladder cancer, but its other molecular mechanisms in the treatment of bladder cancer still need to be further studied.

\section{Acknowledgements}

Not applicable.

\section{Funding}

The present study was supported by Youth Research Project, Health and Family Planning Commission (Fujian, China), 2015 (Grant no. 2015-1-59) and Science and Technology Planning Project, Quanzhou (Fujian, China) 2016 (Grant no. 2016Z044). YL received funding support.

\section{Availability of data and materials}

The datasets used and/or analyzed during the present study are available from the corresponding author on reasonable request.

\section{Author's contributions}

DC designed the study and wrote the manuscript. JC contributed to cell culture. YG made substantial contributions to analysis of data. YL gave the final approval of the version to be published. All authors read and approved the final manuscript.

\section{Ethics approval and consent to participate}

The study was approved by the Ethics Committee of The Second Affiliated Hospital of Fujian Medical University (Quanzhou, China).

\section{Consent for publication}

Not applicable.

\section{Competing interests}

The authors declare that they have no competing interests. 


\section{References}

1. Figueroa JD, Ye Y, Siddiq A, Garcia-Closas M, Chatterjee N, Prokunina-Olsson L, Cortessis VK, Kooperberg C, Cussenot O, Benhamou S, et al: Genome-wide association study identifies multiple loci associated with bladder cancer risk. Hum Mol Genet 23: 1387-1398, 2014

2. Egbers L, Grotenhuis AJ, Aben KK, Alfred Witjes J, Kiemeney LA and Vermeulen SH: The prognostic value of family history among patients with urinary bladder cancer. Int J Cancer 136: 1117-1124, 2015.

3. Chung W, Bondaruk J, Jelinek J, Lotan Y, Liang S, Czerniak B and Issa JP: Detection of bladder cancer using novel DNA methylation biomarkers in urine sediments. Cancer Epidemiol Biomarkers Prev 20: 1483-1491, 2011

4. Tseng-Rogenski S, Gee J, Ignatoski KW, Kunju LP, Bucheit A, Kintner HJ, Morris D, Tallman C, Evron J, Wood CG, et al: Loss of 15-hydroxyprostaglandin dehydrogenase expression contributes to bladder cancer progression. Am J Pathol 176: 1462-1468, 2010.

5. Dong J, Zhai X, Chen Z, Liu Q, Ye H, Chen W and Ling C: Treatment of huge hepatocellular carcinoma using cinobufacini injection in transarterial chemoembolization: A retrospective study. Evid Based Complement Alternat Med: May 17, 2016 (Epub ahead of print). doi:10.1155/2016/2754542.

6. Chen T, Yuan S, Wan XN, Zhan L, Yu XQ, Zeng JH, Li H, Zhang W, Hu XY, Ye YF, et al: Chinese herb cinobufagin-reduced cancer pain is associated with increased peripheral opioids by invaded CD3/4/8 lymphocytes. Oncotarget 8: 11425-11441, 2017.

7. Kau MM, Wang JR, Tsai SC, Yu CH and Wang PS: Inhibitory effect of bufalin and cinobufagin on steroidogenesis via the activation of ERK in human adrenocortical cells. Br J Pharmacol 165 : $1868-1876,2012$

8. Wang ZJ, Sun L and Heinbockel T: Resibufogenin and cinobufagin activate central neurons through an ouabain-like action. PLoS One 9: e113272, 2014.

9. Ma K, Zhang C, Huang MY, Li WY and Hu GQ: Cinobufagin induces autophagy-mediated cell death in human osteosarcoma U2OS cells through the ROS/JNK/p38 signaling pathway. Oncol Rep 36: 90-98, 2016.

10. Lapierre LR, Kumsta C, Sandri M, Ballabio A and Hansen M: Transcriptional and epigenetic regulation of autophagy in aging. Autophagy 11: 867-880, 2015.
11. Zhao L, Tian X, Duan X, Ye Y, Sun M and Huang J: Association of body mass index with bladder cancer risk: A dose-response meta-analysis of prospective cohort studies. Oncotarget 8: 33990-34000, 2017.

12. Franzen CA, Simms PE, Van Huis AF, Foreman KE, Kuo PC and Gupta GN: Characterization of uptake and internalization of exosomes by bladder cancer cells. Biomed Res Int 2014: 619829, 2014. doi: $10.1155 / 2014 / 619829$.

13. Lu XS, Qiao YB, Li Y, Yang B, Chen MB and Xing CG: Preclinical study of cinobufagin as a promising anti-colorectal cancer agent. Oncotarget 8: 988-998, 2017.

14. Yu Y, Wang H, Meng X, Hao L, Fu Y, Fang L, Shen D, Yu X and $\mathrm{Li} \mathrm{J}$ : Immunomodulatory effects of cinobufagin on murine lymphocytes and macrophages. Evid Based Complement Alternat Med 2015: 835263, 2015. doi: 10.1155/2015/835263.

15. Ye M, Qu G, Guo H and Guo D: Specific $12 \beta$-hydroxylation of cinobufagin by filamentous fungi. Appl Environ Microbiol 70: 3521-3527, 2004

16. Wu Q, Lin WD, Liao GQ, Zhang LG, Wen SQ and Lin JY: Antiproliferative effects of cinobufacini on human hepatocellular carcinoma HepG2 cells detected by atomic force microscopy. World J Gastroenterol 21: 854-861, 2015.

17. Yin JH, Zhu XY, Shi WD and Liu LM: Huachansu injection inhibits metastasis of pancreatic cancer in mice model of human tumor xenograft. BMC Complement Altern Med 14: 483, 2014.

18. Salvador-Gallego R, Mund M, Cosentino K, Schneider J, Unsay J, Schraermeyer U, Engelhardt J, Ries J and García-Sáez AJ: Bax assembly into rings and arcs in apoptotic mitochondria is linked to membrane pores. EMBO J 35: 389-401, 2016.

19. Tiwari M, Sharma LK, Vanegas D, Callaway DA, Bai Y, Lechleiter JD and Herman B: A nonapoptotic role for CASP2/caspase 2: Modulation of autophagy. Autophagy 10: 1054-1070, 2014

This work is licensed under a Creative Commons Attribution-NonCommercial-NoDerivatives 4.0 International (CC BY-NC-ND 4.0) License. 